\title{
Calcul des réseaux maillés par l'utilisation de modèles électriques différentiels
}

\section{Interconnected water distribution network analysed by electrical analogy}

\author{
PAR Cr. DUBIN
}

INGÉnieur des ARts et Manufactures

English synopsis p. 636

Pour le calcul des réseaux maillés, il existe, à notre connaissance, deux méthodes qui ont dépassé le stade théorique pour donner un instrument pratique permettant le calcul de réseaux importants :

$1^{\circ}$ La méthode Hardy-Cross (calcul par approximations successives),

$2^{\circ}$ La méthode Camps et HAzEN (calcul par modèle électrique à résistances variables) méthode à laquelle il a été proposé une récente modification par MM. H.-A. Perry, D.-E. Vierling et R.-W. Kohler (Engineering News Record, septembre 1949).

Nous ne reprendrons pas dans le détail la première méthode sur laquelle nous avons publiẻ une étude critique dans la Houille Blanche ( $\mathrm{N}^{\circ} 3$, mai-juin 1947).

Rappelons simplement que nos critiques portaient sur la lenteur de la convergence de cette méthode. Cette lenteur était due au fait que, dans le calcul des approximations successives, la méthode néglige, non seulement des infiniment petits du second ordre, mais également certains infiniment petits du premier ordre.

Le remède proposé consistait à résoudre, non plus une suite de $n$ équations du premier degré à une inconnue ( $\Omega$ : nombre de mailles du réseau) mais un système de $n$ équations à $n$ inconnues.

Cette méthode a été proposée également par M. Malcolm Mc Ilroy dans le Journal Ameri- can Water Works Association de mai 1949, donc deux ans après la publication de notre propre article dont M. Malcolm Mc Ilroy semble ne pas avoir eu connaissance.

D'ailleurs, cette méthode, comme nous l'avons signalé en 1947, a le défaut de ne pouvoir être utilement appliquée qu'à de petits réseaux, car lorsque $n$ devient suffisamment grand, le gain de temps obtenu par l'amélioration de la convergence est largement perdu par la résolution du système de $n$ équations à $n$ inconnues.

Nous n'avons pas parlé jusqu'ici des méthodes électriques, qu'il s'agisse de la méthode Camps et Hazen ou de la méthode plus récente PerryVIERling et Kohler (1). Ces deux méthodes ont. avec la nôtre un point commun : le matériel utilisé est le même; c'est la manière de s'en servir qui est différente. Nous nous réservons de faire un jour, dans la Houille Blanche, une étude comparative de ces méthodes.

Signalons également que, dans l'application pratique de notre méthode, le point de départ (calcul d'un équilibre hydraulique à partir de potentiels arbitraires) est le même que dans la deuxième méthode Hardy-Cross, dite méthode d'égalisation des débits; le principe de cette méthode est exposé dans le mémoire de HARDYCross conjointement à la méthode d'égalisation

(1) Au moment où nous rédigeous cet article, on nous signale également une quatrième méthode exposée dans le dernier numéro du J.A.W.W.A. 
des charges beaucoup plus connue et sur laquelle ont porté tous les commentaires de la méthode Hardy-Cross.

La raison de son peu de succès doit être cherchée dans le fait que telle qu'elle a été exposée dans le mémoire Hardy-Cross, la méthode d'égalisation des débits est, de l'aveu de son auteur, de convergence beancoup plus lente que la première.

Ceci tient à ce que si le calcul des « débits différentiels aux nouds » est correct, le procédé de calcul indiqué pour faire rentrer dans le réseau ces débits différentiels est incorrect.

En effet, Hardy-Cross dit :

- En un nœud $j$ il faut faire rentrer le débit différentiel dans chaque branche proportionnellement à la résistance de cette branche. On voit immédiatement que ceci n'est exact que si tous les nœuds voisins sont au même potentiel, ce qui n'est jamais le cas. Donc, le procédé proposé est complètement arbitraire et ne peut conduire qu'à des déboires.

La mise en équation exacte conduirait ici aussi à un système de $n$ équations à $n$ inconnues ( $n=$ nombre de nœuds) qui ne serait d'ailleurs que la mise en équation de notre réseau différentiel électrique.

Nous avons donc ainsi avec le modèle électrique différentiel une méthode pour résoudre ces $n$ équations sans même les poser.

Cette notion nouvelle, du moins le croyonsnous, car en telle matière, qui peut affirmer sans crainte de démenti être le premier à penser quelque chose, a fait récemment l'objet d'une communication à l'Académie des Sciences. Cette communication a été présentée par notre Président, M. BARRILlon, qui voudra bien nous permettre de lui exprimer ici notre très profonde reconnaissance.

Soit un réseau dans lequel l'écoulement est caractérisé par le système d'équations:

$$
\Sigma \mathrm{Q}_{\mathrm{J}}=0
$$

(qui indique que la somme des débits en un nœud est nulle)

et

$$
\mathrm{U}_{\mathrm{J}}-\mathrm{U}_{\mathrm{K}}=f\left(\mathrm{Q}_{\mathrm{JK}}\right)
$$

(loi d'écoulement dans le conducteur JK).

$\mathrm{Si}$ nous différentions les équations du type (1) et (2) nous trouvons :

$$
\Sigma d \mathrm{Q}_{\mathrm{I}}=0
$$

et

$$
d \mathrm{U}_{\mathrm{J}}-d \mathrm{U}_{\mathrm{K}}=f^{\prime}\left(\mathrm{Q}_{\mathrm{JK}}\right) d Q_{\mathrm{IK}}
$$

Ce sont les lois d'un réseau à loi d'écoulement linéaire admettant :

1. Pour potentiels : les $d U$ du réseau principal;

$2^{\circ}$ Pour débits : les $d Q$ du réseau principal;

$3^{\circ}$ Pour résistance : les valeurs de $f^{\prime}\left(Q_{J K}\right)$ dérivée de la différence de potentiel par rapport au débit.

Ce réseau étant à loi d'écoulement linéaire est donc susceptible d'être représenté par un modèle électrique. Par sa constitution même, ce modèle prend en compte tous les infiniment petits de premier ordre et ne néglige que le second ordre.

Tel est le principe de la méthode par réseaux différentiels.

Mais si nous avons trouvé ce principe du réseau différentiel, il y a bientôt deux ans, nous n'avons pas osé en parler avant d'être sûr que son application pratique donne les résultats théoriquement prévisibles.

Pour pouvoir l'affirmer, il nous manquait l'appareillage électrique et l'expérience de ce genre de mesures.

Fort heureusement pour nous, nous avons trouvé en M. François CAHEN, de la Direction des Etudes et Recherches de l'E.D.F. et en son collaborateur M. MAGNEN, un concours enthousiaste pour mener à bien nos essais.

\section{Utilisation de la méthode}

Considérons un réseau et supposons connus a priori les potentiels aux différents nœuds. L'état de ce réseau est entièrement déterminé puisque les lois d'écoulement nous domnent les débits dans les différents conducteurs et la loi $\Sigma \mathrm{Q}=0$ appliquée en chaque nœud nous donne le débit (entrée ou sortie) extérieur en ce nœud.

Ce que nous cherchons, c'est un état (2) pour lequel les différents débits extérieurs sont donnés, mais dont les potentiels sont inconnus. Si cet état est suffisamment voisin du précédent, une simple différence entre les débits extérieurs de l'état (2) et de l'état (1) nous donne les $d Q$ extérieurs à admettre dans le réseau différentiel dont les résistances sont données par les valeurs des $f^{\prime}\left(Q_{\mathrm{IK}}\right)$ pour l'état (1).

Il suffit donc de mesurer les $d U_{J}$ correspondants sur le réseau électrique et de les ajuster aux $U_{J}$ de l'état (1) pour avoir les $U_{J}$ d'un état $(2)^{\prime}$ celui-ci représentera l'état (2) avec une approximation d'autant meilleure que l'état (1) était plus voisin de l'état (2).

A partir de cet état (2)' nous pouvons recommencer la même opération et ainsi de suite.

Au cours d'expériences faites à l'Electricité de France, nous avons atteint une certaine pré- 
cision fixée à l'avance (erreurs inférieures à $5 \mathrm{~cm}$ sur les hauteurs d'eau aux différents nouds, par exemple) et d'ailleurs très supérieure à celle dont nous nous contenterions en réalité, en cinc approximations successives.

Il est à noter que le calcul par la méthode Haripy-Cross améliorée (améliorations dont nous avons indiqué tout à l'heure les principes) demandait plus de onze approximations successives.

La méthode Hardy-Cross originale en aurait demandé plus de vingt.

Le gain de temps est important surtout si l'on veut bien considérer que le nombre d'erreurs de calculs est très diminué grâce au modèle électrique et grâce à un appareil nomographique pour le calcul des pertes de charge et de leur dérivée dont nous donnons ci-après le principe.

N.D.L.R. - M. MAGNEN, ingénieur des Etudes de l'Electricité de France, a bien voulu faire à la Société Hydrotechnique de France, un exposé personnel sur la réalisation des modèles électriques pour réseaux hydrauliques, notamment pour l'application de la méthode des réseaux différentiels qu'il a mise au point d'après les considérations théoriques de M. DuBn. Cet exposé paraitra incessamment dans la Revue Générale de l'Electricité à laquelle nous renvoyons nos lecteurs que la question intéresserait.

\section{Appareil nomographique pour le calcul rapide des pertes de charge}

Dans un article publié il y a deux ans, nous disions ceci :

« Les approximations successives entraînent des calculs nombreux.

« Il s'agit, pour chaque branche considérée, de calculer les quantités $\mathrm{RQ}^{2}$ et $2 \mathrm{RQ}$.

« Ces calculs s'efrectuent normalement à la règle à ealcul, avee les inconvénients suivants:

«a) Nécessité de consulter un tableau des résistances des différentes branches du réseau pour donner à la réglette la position convenable.

《b) Détermination successive des quantités $\mathrm{RQ}^{2}$ et $2 \mathrm{RQ}$, la règle ne domnant que les chiffres caractéristiques de ces deux quantités, nécessite un effort intellectuel supplémentaire pour la détermination de leur ordre de grandeur.

« La répétition de ces opérations entraîne, à la longue: " $1^{\circ}$ des erreurs de calcul possibles; $2^{\circ}$ une migraine probable de l'opérateur.

«De plus, si au lieu de nous baser, pour la perte de charge, sur une formule en $\mathrm{RQ}^{2}$, nous prenons une formule en $\mathrm{RQ}^{*}$ (Williams HAzkN : $x=1,85$, Flamant : $x=1,75$ ) nous pouvons affirmer que la possibilité du $2^{\circ}$ devient une certitude, même si l'on dispose d'une règle permettant le calcul des puissances fractionnaires, car les règles existant dans le commerce ne permettent lo calcul de $\mathrm{Q}^{x}$ que pour $\mathrm{Q}>1$; si $\mathrm{Q}<1$ le calcul nécessite un double passage par les inverses. $\gg$

Pour pallier ce double inconvénient, nous avions construit un appareil (voir la Houille Blan- che mai-juin 1947) qui n'était autre chose qu'un grand abaque de la fonction :

$$
h=\mathrm{RQ}^{2}
$$

Bien qu'il donnât rapidement la valeur de $h$ avec son ordre de grandeur, on pouvait reprocher à cet appareil son encombrement.

Nous avions d'ailleurs, déjà à l'époque, dessiné les grandes lignes d'un appareil beaucoup moins encombrant. Après les latonnements qu'entraîne toujours la construction d'un prototype, cet appareil a aujourd'hui vu Je jour. Il est en réalité beaucoup plus simple et plus robuste que le premier prototype projeté, car en matière d'invention on va toujours du compliqué au simple.

Cet appareil permet de réaliser n'importe quel abaque à trois alignements parallèles. Il présente un certain nombre d'avantages qu'il n'est pas inutile de signaler.

Les principaux reproches que l'on peut faire aux abaques à alignements viennent de ce que leur précision est limitée:

\section{$1^{\circ}$ Par leurs dimensions.}

En établissant la table à calculer dont nous rous parlions tout à l'heure et qui mesure $2,50 \mathrm{~m} \times 1 \mathrm{~m}$, nous pensons que cela doil à peu près constituer un record en matière d'abaque plan.

$2^{\circ}$ Par le jeu possible du subjectile.

Celui-ci est presque toujours du papier, lequel 
est sensible aux variations de température of d'humidité.

L'appareil que nous avons construit et que nous allons décrire augmente considérablement le champ des abaques à alignements.

Il permet, en effet, de construire des abaques qui occuperaient un plan de $20 \mathrm{~m} \times 10 \mathrm{~m}$. Avec un socle à peine plus volumineux, on pourrait aussi bien envisager des abaques de $100 \mathrm{~m}$ X $50 \mathrm{~m}$ donnant ainsi une précision sensiblement équivalente à celle de la table de logarithme à 5 décimales.

Il est basé sur une idée très simple :

-.- Les abaques ordinaires sont des ahaques à alignements mobiles et échelles fixes.

Nous allons essayer de réaliser, au contraire, un abaque à alignements fixes et échelles mobiles.

Dès lors, les parties des échclles non utilisées pourront être enroulées et on voit qu'il n'y a plus de limite théorique à leur longueur, sinon

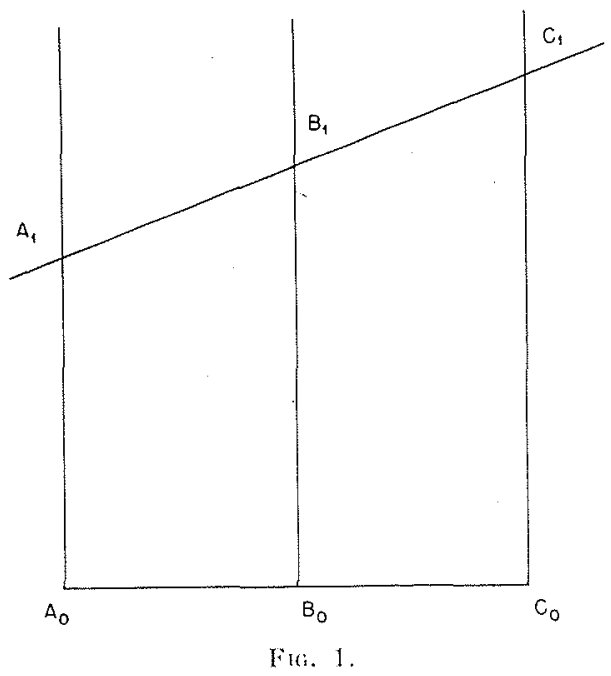

la limite imposée par le temps qu'il faudrait pour dérouler une échelle exagérément longue.

Considérons, sur un abaque à trois échelles parallèles équidistantes, un alignement fixe de base $A_{0} B_{0} C_{0}$ et un alignement quelconque $A_{1} B_{1} C_{1}$. Si nous rendons les échelles mobiles, nous voyons que si l'on ramène indépendamment $A_{1}$ en $A_{0}, C_{1}$ en $C_{0}$, le déplacement de $B_{1}$ en $\mathrm{B}_{0}$ est imposé et a pour valeur :

$$
B_{1} B_{0}=\frac{A_{1} A_{0}+C_{1} C_{0}}{2}
$$

Il est un appareil mécanique bien connu qui donne automatiquement ce déplacement : c'est le différentiel (1).

En effet, si nous considérons un différentiel et si l'on donne une rotation $\omega_{1}$ à un planétaire, $\omega_{2}$ à l'autre planćtaire, l'axe d'un des satellites prend une rotation :

$$
\omega_{3}=\frac{\omega_{1}+()_{2}}{2}
$$

Dès lor's, il suffit de rendre chacun des planétaires et le carter des satellites respectivemenl solidaires de trois tambours sur lesquels seront enroulées les échelles $A_{0} A_{1}, B_{0} B_{1}$ et $C_{0} C_{1}$.

Reste une difficulté pratique:

- Comment réaliser cet enroulement pour qu'il ait lieu sans glissement et à diamètre constant?

Il existe, dans le commerce, un matériel qui permet de réaliser à la perfection la condition imposée : ce sont, d'une part, le film de cinćma et, d'autre part, les tambours d'entrainement pour appareils de projection.

La fixité nécessaire pour l'image à la projection entraîne pour ce matériel une précision d'usinage au $1 / 100^{\circ}$ de $m m$ qui va donc nous donner toute satisfaction.

Dès lors, si l'on veut réaliser, par exemple, l'abaque simple de la multiplication, il suffira de porter sur chacun des films une cehelle logarithmique (simple pour les planétaires, double pour les satellites, si ceux-ci entrainent un lambour de même diamètre que les planétaires, mais on peut tout aussi bien avoir la mème échelle pour le produit que pour les facteurs en dour. blant le diametre du tambour des satellites).

Tel est le principe de l'appareil à trois tambours donnant les mêmes résultats qu'un abaque à trois alignements parallèles.

Dans le cas particulier qui nous occupe, nous avions l'ambition, non seulement d'obtenir la valeur de $R Q^{2}$ comnaissant $R$ et $Q$ mais également la valeur de $2 \mathrm{RQ}$ (disons simplement $\mathrm{RQ}$ puisque les échelles logarithmiques RQ et $2 \mathrm{RQ}$ sont décalées d'une quantité constante : log. 2).

Or, si l'on pose :

on voit que :

$$
\begin{aligned}
& \mathrm{Log} \cdot \mathrm{RQ}^{2}=x \\
& \mathrm{~L} \log \cdot \mathrm{Q}=y
\end{aligned}
$$

$$
\begin{aligned}
& \mathrm{L} \operatorname{Lg} \cdot \mathrm{RQ}^{2}=x+y \\
& \mathrm{Log} \cdot \mathrm{R}=x-y
\end{aligned}
$$

(1) L'application du différentiel comme organe servant à additionner denx longueurs a été déjà utilisé par l'inventeur espagnol Tonnes Y greveno. (Voir Science et Avenir, decembre 1948.) 


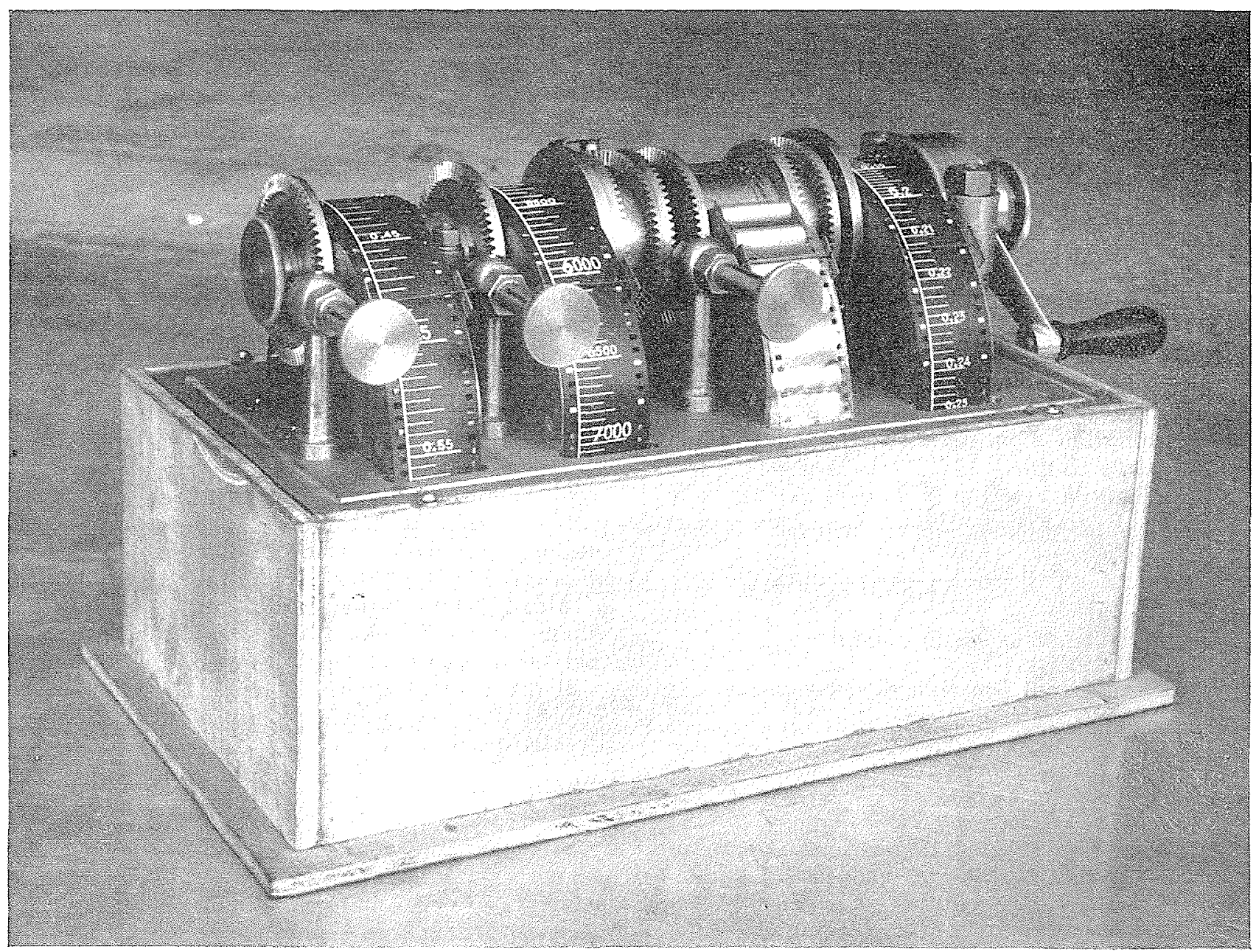

FIG. 2.

A l'appareil précédemment décrit, ajoutons un deuxième différentiel, tel que l'un de ces planétaires soit solidaire d'un planétaire du premier différentiel et subisse ainsi la même rotation $\omega_{1}$, les deux autres planétaires étant solidarisés par un pignon inverseur de telle sorte que le planétaire du deuxième différentiel subisse une rotation - $\omega_{2}$. Ainsi le carter des satellites de ce deuxième différentiel subira une rotation :

$$
\frac{\omega_{1}-\omega_{2}}{2}
$$

Ainsi, nous aurons quatre tambours dont la rotation est respectivement:

$$
\begin{gathered}
\omega_{1} \\
\omega_{2} \\
\frac{\omega_{1}-\omega_{2}}{2} \\
\frac{\omega_{1}-\omega_{2}}{2}
\end{gathered}
$$

II est clair que si nous posons :

$$
\frac{\omega_{1}+\omega_{2}}{2}=x \quad \frac{\omega_{1}-\omega_{2}}{2}=y
$$

Nous aurons:

$\frac{\omega_{1}+\omega_{2}}{2}=x$ tambours des valeurs de $2 \mathrm{RQ}$

$$
\begin{array}{cll}
\frac{\omega_{1}-\omega_{2}}{2}=y & - & \mathrm{Q} \\
\omega_{1}=x+y & - & \mathrm{RQ}^{2} \\
\omega_{2}=x-y & - & \mathrm{R}
\end{array}
$$

Nous aurons ainsi pour les quatre quantités des échelles de même module, donc en tous points semblables.

Pratiquement, nous avons adopté pour unités, de facon à n'avoir plus de virgule dans nos calculs :

- pour les pertes de charge, le cm d'eau, - pour les débits, le litre/seconde. 
Enfin, l'échelle des résistances n'est pas graduée en valeurs des résistances.

Remarquons, en effet, que pour une canalisation donnée, dans un réseau donné, $\mathrm{R}$ est une constante. Il suffit donc de donner à cette canalisation un numéro d'ordre, 15 par exemple, et d'inscrire ce numéro en face d'un repère marqué une fois pour toutes sur l'échelle des résistances et correspondant à la valeur de la résistance de la conduite étudiée. Dans un bureau d'étude comme le nôtre où nous avons à étudier successivement de nombreux réseaux différents, chaque réseau aura ainsi son échelle particulière de résistances que l'on place en quelques secondes sur l'appareil.

Ia graduation d'une telle échelle se fait d'une manière tres simple.

Explicitons la fonction $\mathrm{R}$ d'après une formule comme celle de Scober-Movgne, par exemple :

$$
\mathrm{R}=* \frac{\mathrm{L}}{\mathrm{D}^{5,25}}
$$

où I est la longueur de la conduite,

D son diamètre,

a un coefficient fonction de la rugosité.

On a :

$$
h=\alpha \frac{\mathrm{L}}{\mathrm{D}^{5,25}} \mathrm{Q}^{2}
$$

Si nous prenons un débit $Q=\sqrt{\frac{D^{5,25}}{\alpha}}$ nous voyons que $h=\mathrm{L}$.

Par suite, il suffit de marquer (pour une rugosité \& donnée) sur l'échelle des débits, des repères en face des diverses valeurs de $Q$ correspondant à l'égalité ci-dessus, ces repères étant sradués en diamètres.

Il suffira donc, pour avoir le $R$ correspondant, d'amener le repère de diamètre et de faire marquer la longueur $\mathrm{I}$ de la conduite sur l'échelle des pertes de charge. On marquera alors sur le film des résistances le repère de la canalisation étudiée.

Une échelle de correction pour les différentes rugosités peut être prévue également.

Si l'on ne veut pas surcharger les films, il suffit d'avoir un tableau à double entrée indiquant les débits correspondant aux différents diamètres et rugosités de l'égalité ci-dessus.

Ainsi, nous avons réalisé un appareil qui donne immédiatement et sans ambiguité les pertes de charge et leurs dérivées.

Il est évident qu'un tel appareil peut servir à bien d'autres usages qu'au calcul des pertes de charge.

Mai 1950.

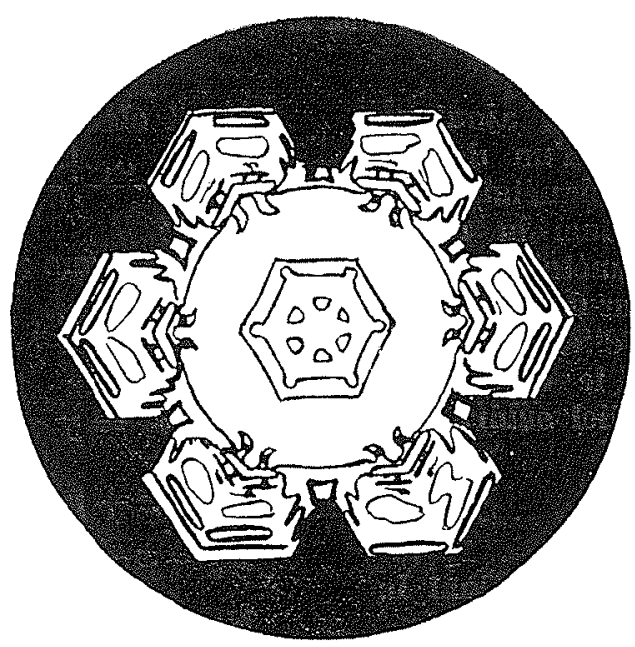

